Marquette University

e-Publications@Marquette

Psychology Faculty Research and Publications

Psychology Department

$3-1-2011$

Nonlinear Dynamical Systems Applications to Psychology and Management

Stephen J. Guastello

Marquette University, stephen.guastello@marquette.edu

Published version. "Nonlinear Dynamical Systems Applications to Psychology and Management," in The SAGE Handbook of Complexity and Management. Eds. Peter Allen, Steve Maguire and Bill McKelvey. Los Angeles: SAGE Publications, 2011: 543-556. Publisher URL: www.sagepub.com/ books/Book233046. (C2011 SAGE Publications. Used with permission. 


\title{
Nonlinear Dynamical Systems Applications to Psychology and Management
}

\author{
Stephen J. Guastello
}

\section{THE BROAD LANDSCAPE OF NDS IN PSYCHOLOGY}

This chapter surveys the recent developments in the application of nonlinear dynamical systems (NDS) theory to theoretical and practical problems encountered in psychology that are also relevant to management. For the benefit of non-psychologists, it is important to note that the scope of psychology is expansive. Introductory textbooks are typically organized around the following themes: brain physiology and behavior, psychophysics, sensation, perception, learning, memory, cognition, intelligence and mental measurement, development, social psychology, motivation and emotion, personality of normal range people, abnormal psychology, psychotherapy and counseling, and industrial-organizational psychology. At the other end of the professional spectrum, the largest professional organization for psychologists, the American Psychological Association, contains more than 50 topical interest groups in addition to its general membership core. The literature on NDS psychology reaches all the major areas of psychology and is growing rapidly (Guastello et al., 2009). For that reason it would be beneficial to focus on the broad themes that have the strongest support at present and that are most relevant to management.

\section{COGNITIVE SCIENCE APPLICATIONS}

Current thinking in NDS theory is that consciousness is an integrated process consisting of psychophysics and sensation processes, perception, cognition, learning, memory, and action. Although it has been convenient to think of these processes as separate entities, the separations are somewhat contrived. An incoming flow of stimuli is first encountered by the human processes of sensation and psychophysical transduction. Perception processes organize the incoming stimuli into recognizable wholes through combinations of learned regimes and innate capabilities. Cognition involves a wide range of processes by which the recognized patterns are compared, associated with information already in memory, transformed in simple or complicated ways, and organized into responses. 
Learning involves one or more processes by which the individual organism acquires knowledge, skills, abilities, and adaptive responses. Memory pertains to how what is learned is organized and stored, and without which learning would be impossible. Psychomotor response - how the response is produced by the individual - is now considered part of an integrated cognition-action process (Shelhamer, 2009). Learning and creativity processes are considered in greater depth next.

\section{Learning}

Learning theory has implications for individual training and development, team building, and the so-called learning organization. Learning theory has undergone numerous developments in psychology in the past century. The major conceptual developments include trial and error learning, the learning curve, the concept of reinforcement, conditioned reflexes and associationism, operant conditioning and schedules of reinforcement, cognitive learning theory and cognitive maps, vicarious learning and imitation. Reinforcement, which proceeded from Thorndike's Law of Effect, depends on reactions from the environment, which in turn developed into an understanding of how information shapes behavior in lieu of actual rewards derived from attaining a behavioral objective.

A more recent regime is implicit learning theory (Seger, 1994), which focuses attention on things that are learned while the learner is trying more deliberately to learn something else. NDS has extended this principle to the explanation of work group coordination, making it a group learning phenomenon (Guastello and Guastello, 1998; Guastello et al., 2005a). Team members implicitly learn to coordinate with each other and entrain their behaviors to each other while engaging in a more explicit task learning objective. Coordination is considered in further depth later in this chapter.

The nonlinear dynamics of learning can follow one of two basic patterns depending on one's interest and emphasis. The first involves chaotic processes leading to selforganization. The learning curve is typically drawn as a smooth function. There is actually a lot of irregularity in the portion of the curve prior to the asymptote (Hoyert, 1992). The neurological explanation is that neural firing patterns are themselves chaotic in the early phases of learning while the brain is testing out possible synaptic pathways. Once learning has progressed sufficiently, the brain locks onto a particular pathway to use consistently (Skarda and Freeman, 1987; Minelli, 2009).

The second dynamic principle involves the cusp catastrophe model. If we extend the baseline of the learning curve (Figure 31.1, left) prior to the onset of the learning trials, two stable states are apparent; according to Frey and Sears (1978) hysteresis exists between learning and extinction curves cannot be explained otherwise. Different inflections in learning curves can be explained as a cusp bifurcation manifold (Guastello et al., 2005a) as shown in Figure 31.1 (right).

The cusp model for the learning process would be

$$
\mathrm{d} y / \mathrm{d} t=y^{3}-b y-a
$$

where control parameter $a$ (asymmetry, governing proximity to the sudden jump) is the ability of a person or the number of learning trials, and control parameter $b$ (bifurcation, size of the sudden jump) would be the difference between treatment and control groups, motivation, or differences in schedules of reinforcement, or any other variable that would contribute to making some learning curves stronger or steeper than others.

The cusp model is particularly good for training and program evaluation. If a statistical cusp effect turns out to be better than the next best alternative linear model it would denote all the features associated with a cusp model. Here the idea of stable end states adds a desirable feature to program evaluation: We want stable improvements to behavior targets, not simply statistically significant differences. 


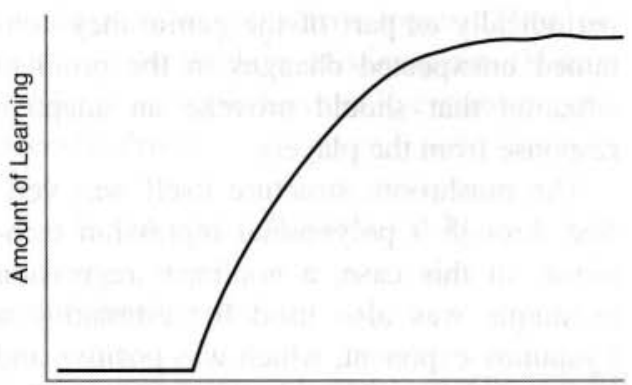

Number of Reinforced Trials

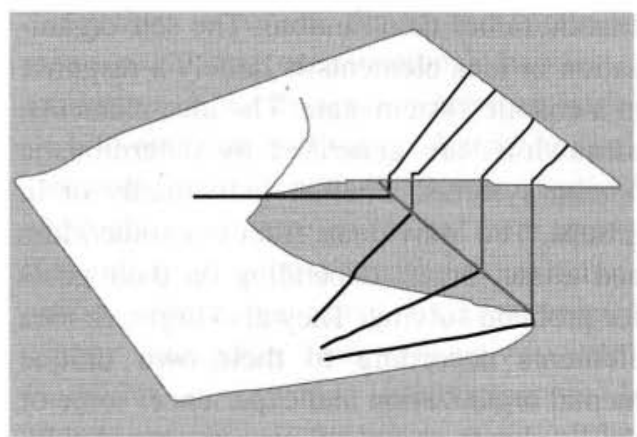

Figure 31.1 Typical learning curve (left) shown as a trajectory on a cusp catastrophe response surface (right). Adapted from Guastello et al. (2005a), with permission of the Society for Chaos Theory in Psychology and Life Sciences

'Stable' does not mean 'without variability', however. A bit of variability is necessary if it will ever be possible for the person, group, or organization to attain greater levels of performance (Abbott et al., 2005; MayerKress et al., 2009). Figure 31.2 illustrates the dynamics of performance improvement. The person, group, or organization encounters a new task that cannot be readily assimilated into old or crystallized learning. With practice the new learning is attained, and the level of hysteresis across the cusp manifold increases with repeated new challenges.

\section{Creative problem solving}

Creativity is a complex phenomenon involving divergent thinking skills, some personality traits that are commonly associated with creative individuals across many professions, an environment rich in substantive and interpersonal resources, and cognitive style. Cognitive style is a combination of personality and cognition; it refers to how people might use their talents rather than the quantity of such talents. According to an early version of the "chanceconfiguration' concept (Simonton, 1988), creative products are the result of a random idea generation process. Greater quantities of ideas are generated from enriched personal and professional environments. Idea elements recombine into configurations as part of the idea generation process. When the creative thinker latches on to a new configuration and explores it as a possible solution to a problem, a form of self-organization of the idea elements takes place.

In the context of NDS, however, the generation and recombination of idea elements is

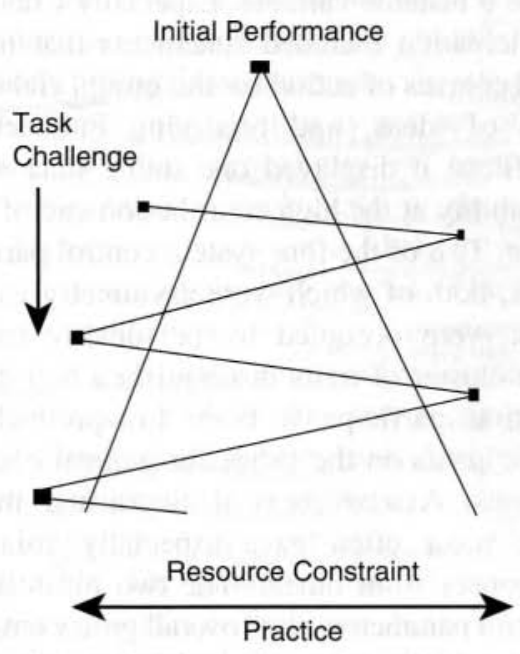

Figure 31.2 Periodic shifts in skill level appear as movements across a cusp manifold 
chaotic rather than random. The self-organization of idea elements is largely a response to a chaotic system state. The idea elements, meanwhile, are generated by deterministic human systems, whether individually or in groups. The individuals filter out some ideas and attract others depending on their goals for problem solving. They also organize idea elements according to their own unique mental organization and experience; some of these mental organizations are shared with other people in the society with other problem solvers in the group, whereas other mental organizations are more unique. The process of idea generation retraces the paths that the individuals have mentally created already among idea elements, prior to any one particular problem-solving event (Guastello, 1995, 1998a).

The mushroom (parabolic umbilic) catastrophe was found to explain the dynamics of creative problem solving in groups who were working together in a real time experiment (Guastello, 1995). The response surface represents two simultaneous and interacting clusters of social interaction patterns. General Participation included information giving, asking questions, and statements of agreement with other people's ideas; it was found to be a bistable variable. Especially Creative Participation included statements that initiated courses of action for the group, elaboration of ideas, and rectifying intellectual conflicts; it displayed one stable state with instability at the high contribution end of the scale. Two of the four system control parameters, both of which were asymmetry variables, were occupied by personality traits. One cluster of traits distinguished high-production participants from low-production participants on the factor for general contributions. Assertiveness distinguished those who most often gave especially creative responses from others. The two bifurcation control parameters were overall group activity level, which captured a social dynamic, and the effect of particular experimental stimuli, which captured an environmental contribution. The news bulletins were introduced periodically as part of the game; they contained unexpected changes in the problem situation that should provoke an adaptive response from the players.

The mushroom structure itself was verified through a polynomial regression technique. In this case, a nonlinear regression technique was also used for estimating a Lyapunov exponent, which was positive and translated into a dimensionality of 5.46. This high dimensionality, which is also fractal, was an important observation because, according to the theory, chaos leads to selforganization, and as creative self-organized systems engender more instability, it would follow that creative problem solving groups are systems operating at the edge of chaos or far-from-equilibrium conditions.

Other studies have also explored whether computer-facilitated communication can enhance the group's overall level of production compared to the production of a collection of noninteracting individuals, so long as the group is large enough to produce a critical mass of ideas. Computer media can facilitate chaotic levels of idea production one would observe bursts of high and low idea production over time by either individuals or groups. Larger changes in production by individuals are associated with greater quantities of ideas that are produced by other group members in between two successive inputs from a particular person. These dynamics conform to the logistic map structure where the contributions by the other group members act as the control parameter (Guastello, 1995).

At the group level of analysis, greater productivity is associated with a relatively complex problem task, where the task can be broken down into subtopics. At that time the group members can work on any subtopic in any order they choose, go back and forth among the subtopics, and so on. In the actual groups studied (Guastello, 1998a), the number of active topics increased and decreased in a periodic fashion. The level of output by the group was chaotic overall, but it also showed periodic rises and drops in activation level in accordance with the change 
in the number of active topics. Thus the result, in the thinking of synergetics (Haken, 1984), is a coupled dynamic consisting of a periodic driver

$$
A_{2}=0.75 A_{1} \exp \left(-0.36 A_{1}\right)+0.33
$$

and a chaotic slave

$$
Z_{2}=\exp \left(0.25 Z_{1}\right)+0.43 A_{1}-0.26 C-0.34
$$

In Eqs (2) and (3), $Z_{1}$ represents group production levels can be observed depending on the topic that the group is working on $(C)$; and $A$ is the number of active discussion threads during the time interval of $Z_{1}$; time was measured in four-day periods. The exponent in Eq. (2) was negative, and the exponent in Eq. (3) was positive.

\section{SOCIAL AND ORGANIZATIONAL PSYCHOLOGY}

This group of topics includes social cognition, motivation, conflict, creative problem solving, group coordination, and leadership emergence. The theory related to motivation extends to a model for personnel selection and turnover, and an interpretation of motivational flow.

\section{Motivation}

Psychological theories of motivation have taken many forms over the years. Hunger and thirst predispose animals to behave as desired in learning experiments. The rat knows where the cheese is, however, we can leap quickly to expectancy theories of motivation whereby the decision maker chooses behavior options that will produce the desired expected reward levels. There is also a theory of equity, in which the agent takes action to restore or maintain equity with other agents.
Another important theme that pervades many social and organizational theories of motivation is the distinction between intrinsic and extrinsic motivation. Extrinsic motivation and extrinsic reward describe situations where the agent receives reward from an outside source. It contrasts with intrinsic motivation, where the agent receives reward, usually intangible, from the activity itself. Examples of intrinsic motivation would include the motives for achievement, affiliation, and power.

Physiological motivation consists of only one form, which is arousal. Arousal originates in the reticular formation of the brain, transfers to the thalamus, and transfers again to the cortical areas where it is interpreted. The same essential process applies to emotion as well.

The butterfly catastrophe model of motivation in organizations draws together many of the previously-known dynamics affecting personnel selection and training, motivation, and work performance, absenteeism, and turnover (Guastello, 1981, 1987, 1995). The principles of several motivational theories are represented in the model. The butterfly catastrophe model consists of three stable states of performance and four control parameters. The three stable states are (a) high performance and initiative, low absenteeism, and low probability of turnover; (b) adequate performance, absenteeism is not out of the norm, and low probability of turnover; (c) performance is inadequate, or absenteeism is excessive, turnover is likely by either voluntary or involuntary means. The four control parameters are ability (asymmetry), extrinsic motivation (bifurcation), intrinsic motivation (swallowtail), and a management climate that tolerates individual differences and encourages intrinsic motivation to dominate over extrinsic motivation (butterfly). The gradients on the butterfly responses surface that run between the stability points and the point of degenerate singularity are interpretable as approach and avoidance gradients in motivation and conflict theory. 
differences in stability versus turbulence in the time series across the 20 people and the three measurements. As a rule, self-efficacy beliefs were relatively stable over time, while motivation and instrumentality were much more volatile.

In a subsequent study (Ceja and Navarro, 2008) participants provided ratings of the same three variables plus others involving challenges and skills at random intervals for 21 days, 6 samples per day. All variables showed deterministic chaos over time, as determined by visual recurrence analysis and comparisons with surrogate data. It was not entirely clear what contributed to the levels of volatility in the latter two studies, although the irregular time intervals could have been responsible.

\section{Conflict}

The available studies on conflict and NDS involve agent-based models, the pathways to chaos as pathways to conflict, or the cusp catastrophe once again as an explanation for approach and avoidance gradients or group polarization. Agent-based models illustrate how individuals working in their own selfinterest produce self-organized systems as they interact with other individuals. Selforganized systems often manifest sudden and discontinuous changes that are recognized as catastrophes or phase shifts (Guastello, 2002). Competition-cooperation dynamics are often inherent in those dynamics (Maynard-Smith, 1982; Axelrod, 1984). They are also inherent in group performance dynamics which are considered in a later section of this chapter.

There are three basic pathways by which a system can become chaotic. The first is an application of the three-body problem. Figure 31.3 shows a more complicated example (from Borges and Guastello, 1996; Guastello, 2002, 2009a) of an attractor field with three attractors (A1, A2, A3) of different strengths. The points labeled $\mathrm{S}$ are saddles, or compromise points between each pair of competing attractors. The opportunity for conflict here is that, if a point enters the field, it is pulled in different directions in an unpredictable way, as denoted by the tangled thread. A partial solution to the conflict between two attractors, which represent two arguable positions on an issue, would define

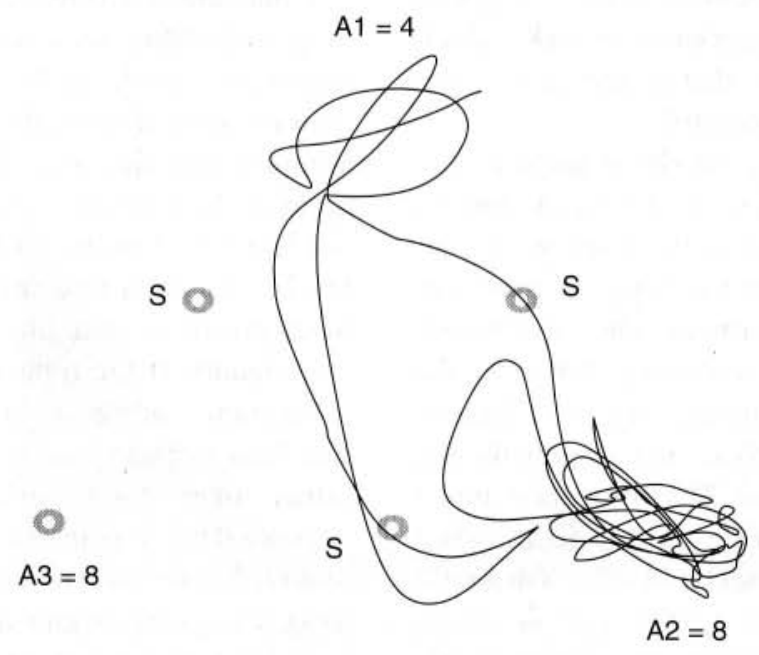

Figure 31.3 Path of a point in a field of three attractors and three saddles. From Guastello (2009a), reprinted with permission of ISCE Publishing 
saddles that could attract points from two directions. In the simulation, a point indicating the position of the solution entered the field in the neighborhood of A1, visited two of the compromise positions, and landed on A2. The implication, nonetheless, is that bilateral agreements are not going to resolve any conflict if there are three or more interest groups involved; $\mathrm{A} 3$ received virtually no attention even though it was as strong as A2. The odds of people changing their preferences for possible solutions often increases as the number of options and the number of interest groups increases. In fact, chaos is more or less guaranteed if there are four participants and four options (Rand, 1978).

The second pathway involves coupled oscillators. Imagine a set of three pendula that are pinjointed together at the ends. When Pendulum 1 oscillates, Pendulum 2 moves faster and its motion pattern becomes more complex than strictly periodic, and Pendulum 3 swings chaotically. The opportunity for conflict can be found in a coupled system involving, for instance, three organizations in a supply chain. Pendulum 3 does not like. being jerked around, and probably cannot function well with all the entropy or unpredictability associated with the motion of the system it is experiencing. In human terms, the uncertainty associated with entropy is equivalent to the experience of risk, which the people or groups that reside later in the chain would like to control.

The third pathway to chaos involves the logistic map bifurcation where a control parameter that increases the level of entropy in the system. When the value of a control parameter passes a critical value, the system oscillates instead of remaining stable. As the value increases further, the oscillations become more complex, and eventually the system goes into chaos. The bifurcation model was a popular concept in organizational development (Michaels, 1989; Guastello et al., 1995; Guastello, 2002). At low values of the control parameter, the system is initially stable (Period 1). Pressure to change (control parameter) has no effect on the system's behavior until the control parameter exceeds a critical threshold. At that point the system oscillates between its old behavior pattern and a new one (Period 2). In the period-doubling regime we would observe the system making complex shifts among multiple behavior patterns. When the system enters chaos (Period 3), the communication and work flows become very inconsistent from moment to moment, or event to event. At this stage the system can self-organize into a new stable pattern and regain its stability by using the new pattern. On the one hand, the bifurcation mechanism explains how to unravel an otherwise stable system in order to make some needed changes. It also characterizes a group exploring ideas for change that could be opposites of each other. Eventually one would need to reverse the control parameter to bring the system back to stability.

Organizational development scenarios often present conflict opportunities because the pressure to change points in one direction while resistance leads to actions that prevent or nullify the change initiative. Although the organizational change agents imagine that the new processes that they are touting are inherently good, that is not necessarily something to be assumed. The complex adaptive system naturally prevents invasive changes from taking root.

Polarization is often connected to conflict in groups, either as a starting point, or as a high-water mark of the group's activities. Groups often discuss their ideas, plans, and attitudes and find they have differences of opinion. In cases where the participants are not too emotionally involved at a personal level, they often find midpoints or compromise positions that are agreeable to most participants. If the topic or attitude target is 'important', however, continued discussion will lead to polarization of group members, rather than compromise. Latane (1996) expressed the dynamics as a cusp catastrophe model. There are two stable states (attractors). The group begins at the unstable point (a saddle) on the surface and then splits into distinctive poles if the importance of the attitude is high, and does not polarize for less important attitudes. 
In a related theme, Vallacher et al. (2010) characterized intractable conflict states themselves are single attractors. The attractors are formed by combinations of attitudes, goals, and more importantly, interaction patterns among the conflicting parties. If a situation is inherently complex there are some elements that are salient and closely linked to some of the other elements, but could also be some elements that are not linked or attended to as well as they should be linked. The unattended elements self-organize into a latent attractor that presents conflict with the more manifest attractor. The boilerplate solution to conflict is to break up some of the interaction patterns, thus creating entropy and a search for a new attractor where elements are connected differently, perhaps in a more integrated fashion, and presumably life would be better.

\section{Leadership emergence}

The rugged landscape model of self-organization offers a cogent explanation for organizational phenomena, particularly where strategic management is involved (McKelvey, 1999). The rugged landscape model of self-organization also explains how leaders emerge from a leaderless group, and the possible ways in which their emergence could take form
(Guastello et al.,. 1998b, 2007a, b; Zaror and Guastello, 2000; Guastello et al., 2005b). The group activity selected for study involved a complex creative problem solving task. Once presented with the task and an hour (of experimental time) to complete it, numerous verbal interactions transpire among group members. These local interactions culminate in the eventual self-organization of the group such that the role of a general leader emerges along with several other, more specific roles.

The formation of roles would constitute fitness peaks, which denote relative fitness, local stability, and clusters of similar subspecies with regard to shared adaptive traits. The probability density function that is associated with the swallowtail catastrophe model (Eq. (4), Figure 31.4) describes the distribution of people into unstable and locally stable social roles. The swallowtail catastrophe structure contains a response surface of discontinuous events, or qualitatively different outcomes, such that there are two stable states, with a minor antimode between them, an unstable state, and a major antimode separating the unstable state from the two stable ones:

$$
\begin{aligned}
\operatorname{Pdf}(z)= & \xi \exp \left(\theta_{1} z^{5}+\theta_{2} z^{4}+\theta_{3} c z^{3}\right. \\
& \left.+\theta_{4} b z^{2}+\theta_{5} a z\right)
\end{aligned}
$$

In Eq. (4), $z$ is the extent to which members of the group endorse a particular group

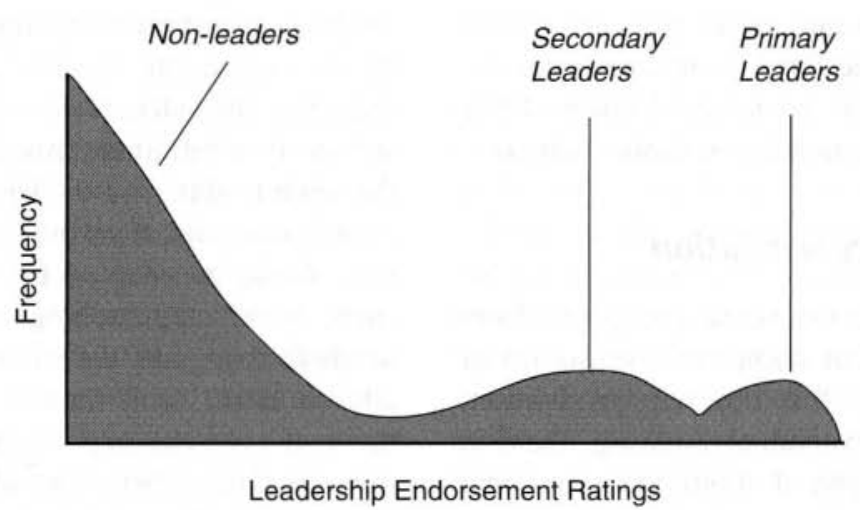

Figure 31.4 Swallowtail catastrophe distribution of leadership ratings after a leadership emergence process. From Guastello (2007b), reprinted with permission of the American Psychological Association 
Table 31.1 Summary of results from leadership emergence studies with the swallowtail catastrophe model*

\begin{tabular}{|c|c|c|c|}
\hline Type of group & Asymmetry & Bifurcation & Swallowtail \\
\hline $\begin{array}{l}\text { Creative problem } \\
\text { solving }\end{array}$ & $\begin{array}{l}\text { General participation and control of the } \\
\text { conversation; including gate-keeping, } \\
\text { initiating, following, harmonizing, } \\
\text { facilitating the ideas of others, task } \\
\text { orientation, consideration of other } \\
\text { players' interests, concern for solution } \\
\text { quality. }\end{array}$ & $\begin{array}{l}\text { Giving information, creative } \\
\text { ideas, competitive behavior, } \\
\text { concern for solution quality. }\end{array}$ & Unknown \\
\hline Production & $\begin{array}{l}\text { Tension reduction, including } \\
\text { harmonizing, giving information, goal } \\
\text { realism. }\end{array}$ & $\begin{array}{l}\text { Creative and task control, } \\
\text { controlling the conversation. }\end{array}$ & Unknown \\
\hline $\begin{array}{l}\text { Coordination- } \\
\text { intensive }\end{array}$ & $\begin{array}{l}\text { General participation and control of the } \\
\text { conversation; including gatekeeping, } \\
\text { initiating, following, creative ideas, } \\
\text { facilitating the ideas of others. }\end{array}$ & $\begin{array}{l}\text { Verbal vs. non-verbal } \\
\text { working conditions }\end{array}$ & Task control \\
\hline $\begin{array}{l}\text { Emergency } \\
\text { response }\end{array}$ & $\begin{array}{l}\text { Wide-range competitive behavior against } \\
\text { adversary, controlling the moves of the } \\
\text { team, helped other members make good } \\
\text { moves, asked questions, contributed } \\
\text { information, boosted team morale. }\end{array}$ & Group size & Group performance \\
\hline
\end{tabular}

* Summarized from Guastello et al. (2005b), Guastello and Bond (2007a), Guastello (2010a).

member as the leader; $a, b$, and $c$ are control parameters; $\xi$ is a constant that maintains unit density; and $\theta_{i}$ are nonlinear regression weights. The model requires three control parameters. Research to date has investigated the nature of the control variables, which vary in their content depending on what type of group is involved, e.g. creative problem solving, production, and coordination-intensive groups. One control parameter $(a)$ distinguishes all leaders from non-leaders. The second $(b)$ controls the extent to which the leaders stabilize into either primary or secondary roles. The third $(c)$ distinguishes the primary from the secondary leaders. Table 31.1 contains a summary of those findings.

\section{Work group coordination}

Coordination occurs when group members make the same or compatible responses at the right time for optimal production. Contrary to conventional thinking, there is more than one type of coordination in game theory. As with any type of game, individuals make decisions based on the utilities associated with the options. Prisoner's Dilemma involves choices between cooperation and competition. The Stag Hunt game involves choices between joining the group (to hunt stag) and going off on one's own (to hunt rabbits). A potential negative outcome in Stag Hunt is social loafing or the free rider syndrome.

The Intersection game requires group members to take the correct actions in the correct sequence, and to figure out the correct sequence, similar to what occurs in a four-way stop intersection. If the drivers correctly perceive the turn-taking system adopted by the preceding drivers and follow the sequence, then all cars pass through the intersection in a minimum amount of time with the lowest odds of a collision. In a real-life intersection, any of several possible rule systems could be adopted by the drivers, and each driver approaching the intersection needs to recognize the strategy that is actually in effect, and then make the correct move. If a car tries to go through the intersection out of turn, then an accident could occur, or at the very least, other players would need to revert to ad lib turn-taking to untangle the confusion at the intersection. 
The process of group coordination involves the development of nonverbal communication links among the participants. These links evolve with repeated practice with each other. The evolution of the links is essentially a self-organization process. Furthermore, the basic process of coordination is non-hierarchical, meaning that a leader, who usually contributes task structuring activities of some sort, is not required. This state of affairs is not unlike the flocking of birds, herds of beasts, or schools of fish, which operate without leaders.

The results of Intersection game experiments to date show that if the experimental task is not excessively difficult, the group will display a coordination learning curve (Guastello and Guastello, 1998). The coordination acquired during one task session will transfer to the learning and performance curve of a second task. If the task is too difficult, self-organization will not be complete, and the time series of coordination data will be chaotic. A coordinated group can withstand changes in personnel up to a point before coordination breaks down (Guastello et al., 2005b). Verbalization enhances performance to some extent, but not necessarily the level of leadership emergence (Guastello and Bond, 2007a).

\section{Coordination and hierarchies}

Coordination does not require leaders, and the mainstay of game theory experiments in economics are conducted without leaders or even talking between the participants (Friedman, 1994). One premise of evolutionary game theory is that a large volume of simple bilateral interactions produces global results for the social system. Individuals can adopt hierarchical rules or strategies (oligarchic reaction functions) such as tit-for-tat. Again, leaders and hierarchical relationships are not necessary (which explains some of game theory's popularity with neo-classical economists). Another key point is that the relationship between long-run equilibria (evolutionarily stable states) and the utilities within single-shot games is not always consistent.

The forms of coordination observed in non-hierarchical non-human species are not leader-follower relationships. A flock of birds will stick together on the basis of only three rules: following the general heading of the flock, stick close to the flock, and do not crash into flock mates. The goose at the vertex of a V formation is not the leader; they rotate positions. A school of fish stick together in much the same way; they have a rule of motion whereby they exchange positions from the outside to the inside of the school and out again as a means of hedging against predators. Wilson (1975) suggested that leadership occurs in non-hierarchical groups when one member of the flock detects a predator first, even if by virtue of keener sight or smell, or a more advantageous location for detecting signals. The animal that moves first moves the group. The member of the flock who has keener senses, or flies fastest, moves the group most often and appears most similar to anthropomorphic leaders.

Southeast Asian fireflies will start the evening by flickering quasi-randomly, but after a few hours they synchronize into a coordinated pulse throughout the forest. Synchronicity can be produced even in nonliving systems with only minimum requirements - two coupled oscillators, a feedback channel between them, and a control parameter that speeds up the oscillations (Strogatz, 2003). The oscillators synchronize when they speed up fast enough. The principle also has been demonstrated with electrical circuits and mechanical clocks. Leadership is irrelevant to circuits and clocks.

None of the above negates the principle that leaders can emerge in coordination-intensive human task groups that begin without leaders. Members that do emerge as leaders exhibit a wide range of behaviors that are useful to the group who can communicate freely and exert control over the task. Thus they become the hub of communication (information flow) in both verbal and nonverbal modalities (Guastello and Bond, 2007b). 
So what do leaders actually do that is constructive? Leaders can invent options for goals and means of attaining them. Leaders can alter perceptions of utilities, and a good sense of reality is critical here. Leaders can become the hub of communication. Leaders can set the pace for the group's work.

There is some agreement (Guastello, 2008; Van Vugt et al., 2008), nonetheless, that leadership is not necessary for many types of tasks, and that constituents can adopt strategies to influence the behavior of leaders. Nonlinear dynamics offers a more direct path to the same conclusions, however, and with additional insights: Emergent group structures and performance patterns can form strictly from the bottom up with or without a supervenience principle whereby the upper level dominates the actions of the lower level. It is overly simplistic to think that the upper level dominates the lower and that is the end of the story. Experimental evidence shows that the antics of the lower level can destabilize performance at the upper level, and the skill of managing a workflow within a hierarchy is not widely shared (Guastello, 2002: Chapter 10). Even in the most benign case where people are just trying to do their jobs, management can be very scattered in its efforts to stabilize a work flow. At present it is not clear how much of the skill for managing this form of chaos could be trainable, or something to be studied in a personnel selection context.

\section{FUTURE DIRECTIONS}

Empirical verification is always an issue in psychology generally, not only in NDS applications. Dooley (2009) observed that empirical studies of NDS in organizational behavior that involve real data are rare, particularly in comparison to the number of well-reasoned concept pieces that have been written. The logistic map model for organizational change, for the times it has been cited as a prototype of the change process, has not received any direct empirical study.
Empirical analysis is nowhere near impossible as the studies captured in this chapter have illustrated. One does not need a godzillion data points to assess a fractal dimension or any other important dynamical indicator, nor is it necessary to test a myriad dynamical models devised by mathematicians to determine a viable model for real-world data (Gregson and Guastello, 2005; Guastello, 2009b; Guastello and Liebovitch, 2009). Techniques built on the generic characteristics of chaos, self-organization and other dynamics, such as entropy measurements and structural statistical equations, serve the purposes well.

By the same token, many of the theoretical models in this chapter have been empirically demonstrated only once, although a few have received more attention. It would appear that significant and practical advances can be made by building on NDS models that are known already concerning learning, creative problem solving, motivation, personnel selection, leadership emergence, work group coordination, and work flows in hierarchies. The material on conflict in organizations is relatively new, however. The principles of pathways to chaos are internally rigorous, yet it would be beneficial to see how they play out during real-world conflict resolution projects.

At the theoretical level of development, the concept of the complex adaptive system is central to our understanding organizations. Psychology has begun to consider what adaptive behavior could look like (Pulakos et al., 2000). There is a sense that learning and creative behavior are both involved. It would follow that a highly functional theory could result from building on the known dynamics of learning and creativity, and make greater use of NDS indicators of turbulence and adaptation such as the Lyapunov exponent (Guastello, 2010b).

\section{REFERENCES}

Abbott, A., Button, C., Pepping, G-J., and Collins, D. (2005). Unnatural selection: Talent identification 
and development in sport. Nonlinear Dynamics, Psychology, and Life Sciences, 9: 61-88.

Axelrod, R. (1984). The Evolution of Cooperation. New York: Basic Books.

Borges, L.A.J. and Guastello, S.J. (1996). Integration and strategy: A chaos theory approach. In: C.A. Voss (ed.) Manufacturing Strategy: Operations Strategy in a Global Context. London, UK: London Business School, pp. 97-102.

Ceja, L. and Navarro, J. (2008). Dynamics of flow: A nonlinear perspective. Journal of Happiness Studies: DOI 10.1007/s.10902-008-9113-6.

Csikszentmihalyi, M. (1990). Flow: The Psychology of Optimal Experience. New York: Harper-Collins.

Dooley, K.J. (2009). Organizational psychology. In: S.J. Guastello, M. Koopmans, and D. Pincus (eds) Chaos and Complexity in Psychology: Theory of Nonlinear Dynamical Systems. New York: Cambridge University Press, pp. 434-451.

Frey, P.W. and Sears, R.J. (1978). Model of conditioning incorporating the Rescorla-Wagner associative axiom, a dynamic attention process, and a catastrophe rule. Psychological Review, 85: 321-340.

Friedman, J.W. (ed.) (1994). Problems of Coordination in Economic Activity. Boston: Kluwer.

Gregson, R.A.M. and Guastello, S.J. (2005). Introduction to nonlinear methodology, part 1: Challenges we face and those that we offer. Nonlinear Dynamics, Psychology, and Life Sciences, 9: 371-374.

Guastello, S.J. (1981). Catastrophe modeling of equity in organizations. Behavioral Science, 26: 63-74.

Guastello, S.J. (1987). A butterfly catastrophe model of motivation in organizations: Academic performance. Journal of Applied Psychology, 72: 165-182.

Guastello, S.J. (1995). Chaos Catastrophe and Human Affairs. Mahwah, NJ: Erlbaum.

Guastello, S.J. (1998a). Creative problem solving groups at the edge of chaos. Journal of Creative Behavior, 32: 38-57.

Guastello, S.J. (1998b). Self-organization and leadership emergence. Nonlinear Dynamics, Psychology, and Life Sciences, 2: 303-316.

Guastello, S.J. (2002). Managing Emergent Phenomena: Nonlinear Dynamics in Work Organizations. Mahwah, NJ: Lawrence Erlbaum Associates.

Guastello, S.J. (2007a). Nonlinear dynamics and leadership emergence. Leadership Quarterly, 18: 357-369.

Guastello, S.J. (2007b). How leaders really emerge. American Psychologist, 62: 606-607.

Guastello, S.J. (2008). Evolutionary game theory and leadership. American Psychologist, 64: 53-54.

Guastello, S.J. (2009a). Chaos and conflict: Recognizing patterns. Emergence: Complexity in Organizations, 10(4), 1-9.
Guastello, S.J. (2009b). Chaos as a psychological construct: Historical roots, principal findings, and current growth directions. Nonlinear Dynamics, Psychology, and Life Sciences, 13: 289-310.

Guastello, S.J. (2010a). Self-organization and leadership emergence in emergency response teams. Nonlinear Dynamics, Psychology, and Life Sciences, 14: 179-204.

Guastello, S.J. (2010b). Nonlinear dynamics of team performance and adaptability in emergency response. Human Factors, 52, DOI: 10.1177/00187 20809359003.

Guastello, S.J., Dooley, K.D., and Goldstein, J. (1995). Chaos, organizational theory, and organizational development. In: F.D. Abraham and A. Gilgen (eds) Chaos Theory in Psychology, Westport, CT: Greenwood Publishing Group, pp. 267-278.

Guastello, S.J. and Guastello, D.D. (1998). Origins of coordination and team effectiveness: A perspective from game theory and nonlinear dynamics. Journal of Applied Psychology, 83: 423-437.

Guastello, S.J., Johnson, E.A., and Rieke, M.L. (1999). Nonlinear dynamics of motivational flow. Nonlinear Dynamics, Psychology, and Life Sciences, 3: 259-274.

Guastello, S.J., Bock, B., Caldwell, P., and Bond, R.W. Jr. (2005a). Origins of group coordination: Nonlinear dynamics and the role of verbalization. Nonlinear Dynamics, Psychology, and Life Sciences, 9: 175-208. Guastello, S.J., Craven, J., Zygowicz, K.M., and Bock, B. R. (2005b). A rugged landscape model for self-organization and emergent leadership in creative problem solving and production groups. Nonlinear Dynamics, Psychology, and Life Sciences, 9: 297-333.

Guastello, S.J. and Bond, R.W. Jr. (2007a). The emergence of leadership in coordination-intensive groups. Nonlinear Dynamics, Psychology, and Life Sciences, 11: 91-117.

Guastello, S.J. and Bond, R.W. Jr. (2007b). A swallowtail catastrophe model of leadership in coordinationintensive groups. Nonlinear Dynamics, Psychology, and Life Sciences, 11: 235-351.

Guastello, S.J. and Liebovitch, L.S. (2009). Introduction to nonlinear dynamics and complexity. In: S.J. Guastello, M. Koopmans, and D. Pincus. (eds) Chaos and Complexity in Psychology: Theory of Nonlinear Dynamical Systems. New York: Cambridge University Press, pp. 1-40.

Guastello, S.J., Koopmans, M., and Pincus, D. (eds) (2009). Chaos and Complexity in Psychology: Theory of Nonlinear Dynamical Systems. New York: Cambridge University Press.

Haken, H. (1984). The Science of Structure: Synergetics. New York: Van Nostrand Reinhold. 
Hoyert, M.S. (1992). Order and chaos in fixed-interval schedules of reinforcement. Journal of the Experimental Analysis of Behavior, 57: 339-363.

Latane, B. (1996). Dynamic social impact: The creation of culture by communication. Journal of Communication, 46: 13-25.

McKelvey, B. (1999). Avoiding complexity catastrophe in coevolutionary pockets: Strategies for rugged landscapes. Organization Science, 10: 294-321.

Mayer-Kress, G., Newell, K.M., and Liu, Y-T. (2009). Nonlinear dynamics of motor learning. Nonlinear Dynamics, Psychology, and Life Sciences, 13: 3-26.

Maynard-Smith, J. (1982). Evolution and the Theory of Games. Cambridge, UK: Cambridge University Press.

Michaels, M.D. (1989). The chaos paradigm. Organizational Development Journal, 7(2): 31-35.

Minelli, T.A. (2009) Neurodynamics and electrocortical activity. In: S.J. Guastello, M. Koopmans, and D. Pincus (eds) Chaos and Complexity in Psychology: Theory of Nonlinear Dynamical Systems. New York: Cambridge University Press, pp. 73-104.

Navarro, J., Arrieta, C., and Ballen, C. (2007). An approach to the study of dynamics of work motivation using the diary method. Nonlinear Dynamics, Psychology, and Life Sciences, 11: 473-498.

Pulakos, E.D., Arad, S., Donovan, M.A., and Plamondon, K.E. (2000). Adaptivity in the workplace: Development of a taxonomy of adaptive performance. Journal of Applied Psychology, 85: 612-624.
Rand, D. (1978). Exotic phenomena in games and duopoly models. Journal of Mathematical Economics, 5: 173-184.

Seger, C.A. (1994). Implicit learning. Psychological Bulletin, 115: 163-196.

Shelhamer, M. (2009). Special issue: psychomotor coordination and control. Nonlinear Dynamics, Psychology, and Life Sciences, 13, 1-2.

Simonton, D.K. (1988). Creativity, leadership, and change. In: R.J. Sternberg (ed.) The Nature of Creativity: Contemporary Psychological Perspectives. Cambridge, MA: MIT Press, pp. 286-426.

Skarda, C.A. and Freeman, W.J. (1987). How brains make chaos in order to make sense of the world. Behavioral and Brain Science, 10: 161-195.

Strogatz, S. (2003). Sync: The Emerging Science of Spontaneous Order. New York: Hyperion.

Van Vugt, M., Hogan, R., and Kaiser, R.B. (2008). Leadership, followership, and evolution: Some lessons from the past. American Psychologist, 63: 182-196.

Vallacher, R.R., Coleman, P.T., Nowak, A., and BuiWrzosinska, L. (2010). Rethinking intractable conflict. American Psychologist, 65: 262-278.

Wiggins, S. (1988). Global Bifurcations and Chaos. New York: Springer-Verlag.

Wilson, E.0. (1975). Sociobiology. Cambridge, MA: Harvard University Press.

Zaror, G. and Guastello, S.J. (2000). Self-organization and leadership emergence: A cross-cultural replication. Nonlinear Dynamics, Psychology, and Life Sciences, 4: 113-119. 